\title{
GROUNDWATER MANAGEMENT AND AGRICULTURE
}

\author{
Marta Biancardi ${ }^{338}$ \\ Lucia Maddalena $^{339}$
}

https://doi.org/10.31410/itema.2018.992

\begin{abstract}
Over the last decades, groundwater irrigation has become commonplace in many regions worldwide. This is largely a consequence of the advances in drilling and pumping technologies and of the development of hydrogeology. Compared with traditional surface water irrigation systems, groundwater irrigation offers more reliable supplies, lesser vulnerability to droughts, and ready accessibility for individual users. However, poor groundwater management and legal controversies are currently at the base of social disputes in the water exploitation. A through and transparent assessment of the relative socio-economic value of groundwater can contribute to mitigate or avoid potential conflicts. In this paper, we develop a mathematical model that studies the exploitation of a common groundwater resource in order to take into account the strategic and dynamic interactions among the users of the resource. It is known that a number of different factors may increase the welfare gains of firms but also cause the overexploitation of the aquifer. The aim of the model is to analyze policy options and economic forces that influence the groundwater irrigation sector and its development.
\end{abstract}

Keywords: Groundwater extraction, Agriculture, differential games.

\section{Introduction}

quifer overexploitation is a serious problem in many regions of the world. The extra
use of this resource has caused, in the last years, water table drawdowns and depletion.

Intensive use of groundwater leads to a wide array of social, economic and environmental consequences such as land subsidence, increases in the vulnerability of agriculture and other uses of the water to climate change, increases in pumping costs, Burke (2003).

The open-access nature of natural resources, such as groundwater and the accompanying externalities, in combination with the failure to treat natural resources as capital, has made this an attractive research area, Brown (2000), for the development of rules for efficient water allocation among competing uses over time and space Koundouri and Xepapadeas (2004).

We consider a groundwater resource used for irrigation by several farmers. Common groundwater resources are often exploited under a common property regime, that is the access is restricted to land owners situated over the aquifer.

Interest in water resources conflict resolution has increased over the last decades and various quantitative and qualitative methods have been proposed for conflict resolution in water resources management. Numerous papers have studied this issue (for example Gisser and Sanchez (1980), Negri (1989), Provencher and Burt (1993), Rubio and Casino (2001, 2003))

\footnotetext{
${ }^{338}$ Department of Economics, University of Foggia, Largo Papa Giovanni Paolo II,1, 71121 - Foggia, Italy

${ }^{339}$ Department of Economics, University of Foggia, Largo Papa Giovanni Paolo II,1, 71121 - Foggia, Italy
} 
and have concluded that private exploitation is inefficient, in terms of stock and welfare, in comparison to optimal exploitation. This inefficiency is due to the various externalities which appear because of the sharing of this type of resource, namely the pumping cost externality which characterizes the fact that withdrawals made by one farmer lower the water-table level, resulting in an increase in pumping costs for the other users. On the other hand, the stock externality, also called strategic externality, represents competition between farmers because of the limited availability of the water stock (see Provencher and Burt (1993)). For an overview of these results, it is possible to see Koundouri (2004). Esteban and Albiac (2011) take ecosystem damages into consideration in modeling aquifer management regimes and they show that by including these environmental externalities into the analytical framework, results can change substantially. Biancardi and Maddalena (2018) analize the case in which firms are divided in cooperators and defectors in the exploitation of the groundwater resource.

In this paper we see that countries are different for their behaviour. The motivation of our work is based on the idea that some exogenous stumbling block are not taken into account in previous studies. The issue of illegal wells is paramount in the aquifer it is not present in all papers quoted. Illegal water use is, in fact, a key issue to understand many of the problems related to depleting and overexploited stocks. Empirical evidence demonstrates that illegal activities, including unlicensed water extraction, illegal logging and pollution discharges, are at the root of the degradation of water-related habitats. FAO (2008) calls attention to the role of agriculture in the deterioration process. Some authors have recently begun to analyze the problem of non-compliance with resource management regimes, based on the literature on social norms in common property resource.

Our extension to existing literature is that we consider heterogeneous farmers in terms of behavior of the exploitation of the water resource. In particular, we consider $\mathrm{N}$ identical firms that differ in terms of their choice to extract in legal or illegal way. It is clear that illegal extractions are a significant stumbling block on the path towards the implementation of better management policies.

We study the exploitation of a common groundwater resource as a differential game in order to take into account the strategic and dynamic interactions between the users of the resource by several farmers. In particular, we analyze open-loop equilibria and we illustrate the implications of the different strategies on extraction rates and groundwater table levels. Results highlight the effects of the penalties on the height of the water and on the quantities of water pumped.

The paper is organized in the following way. Section 2 presents the model, while Section 3 presents the differential game and computes open-loop Nash equilibria. Section 4 proposes a comparative statics about the parameters of the model. Section 5 concludes.

\section{The basic model}

The model proposes a dynamic equation for the water table and a set of net benefits from groundwater use. The differential equation which describes the dynamics of the water table is obtained as the difference between natural recharge and net extractions

$$
\dot{H}=\frac{1}{A S}[R+(\gamma-1) W] \quad H(0)=H_{0}
$$


where $H$ is the height of the aquifer, i.e. the water table elevation above some arbitrary level that is considered as being the bottom of the aquifer, $R$ denotes the natural recharge, $A S$ is the area of the aquifer, $0<\gamma<1$ is the constant return flow coefficient of irrigation water and $W$ is the amount of the groundwater pumped.

Access to the aquifer is restricted by land ownership and consequently the number of farmers is fixed and finite over time. We assume that $N$ is the number of farmers and that they are divided in two groups: $m$ farmers who have a permit and pump legal water and are said legal farmers and $N-m$ farmers who are not authorized to pump but still do it they are said illegal farmers. Let $w_{l}(l=1, \ldots, m)$ and $w_{i}(i=m+1, \ldots, N)$ the pumping rate of the legal and illegal farmers so that the amount of groundwater pumped is

$$
\sum_{l=1}^{m} w_{l}+\sum_{i=m+1}^{N} w_{i}=W
$$

The net benefits obtained from production by a given farmer $k$ are

$$
\pi_{k}=F\left(w_{k}\right)-C\left(w_{k}, H\right)
$$

where $F\left(w_{k}\right)$, for $k=i$ or $k=l$, is the benefit of water use and $C\left(w_{k} ; H\right)$ is the cost of water pumping, which depends on the amount of water pumped by the user, $w_{k}$, as well as on the height of the water table $H$, because as the water table sinks deeper, pumping costs should increase. Regarding production benefit we have that:

- The marginal benefit of using water is non-negative but decreasing, i.e.

$$
\frac{\partial F}{\partial w} \geq 0 \text { and } \frac{\partial^{2} F}{\partial w^{2}} \leq 0
$$

Regarding pumping costs the following properties are true:

- The marginal cost of pumping water is non-negative and increasing, i.e.

$$
\frac{\partial C}{\partial w} \geq 0 \text { and } \frac{\partial^{2} C}{\partial w^{2}} \geq 0
$$

- The effect of the water table height can be summarized by

$$
\frac{\partial C}{\partial H} \leq 0 \quad, \quad \frac{\partial^{2} C}{\partial H^{2}} \geq 0 \quad \text { and } \frac{\partial^{2} C}{\partial w \partial H} \leq 0
$$

The benefit functions for illegal farmers are affected by penalties associated with illegal behavior. They are expressed by the following term

$$
P\left(w_{i}, \phi, f\right)
$$


where the enforcement intensity $0 \leq \phi \leq 1$ is the probability of catching illegal firms and $f \geq 0$ is the level of penalty. In order to find the closed form equilibrium, we refer to a specific functional form of benefits and costs. The net revenues of a legal firm are equal to the willingness-to-pay for groundwater minus the extraction costs of the resources

$$
\pi_{l}=\frac{-\beta}{2} w_{l}^{2}+\alpha w_{l}-\left(c_{0}-c_{1} H\right) w_{l}
$$

The net revenues of an illegal firm are equal to the willingness-to-pay for groundwater minus the extraction costs of the resources and minus penalties

$$
\pi_{i}=\frac{-\beta}{2} w_{i}^{2}+\alpha w_{i}-\left(c_{2}-c_{1} H\right) w_{i}-\phi f w_{i}
$$

where $\alpha>0$ and $\beta>0$ are constants that describe the effects of irrigation on the crop, the fixed costs are $0<c_{2}<c_{0}$ instead the marginal pumping cost per acre foot of water pumped per foot of lift is $c_{1}>0$, equal for legal and illegal countries.

\section{The differential game}

In the optimal control problem, the objective of each country is to maximize its discounted profit. We propose a differential game, in which we calculate open-loop Nash equilibria in order to determine both the optimal paths of the extraction levels and the dynamic of the water table. Time $t$ is continuous, with $t$ in $[0,+\infty[$ and countries discount future costs using the constant rate $r>0$. We use a non-cooperative game framework solved in a backward order. As result of the first stage, $m$ legal firms and $N-m$ illegal ones exist and, in the second stage, firms choose their pumping levels.

Fixed the pumping levels of illegal countries, legal firms bind to a level of extraction that maximizes the discounted value of their payoff:

$$
\pi_{l}=\max _{w_{l}} \int_{0}^{+\infty} e^{-r t}\left\{\frac{-\beta}{2} w_{l}^{2}+\alpha w_{l}-\left(c_{0}-c_{1} H\right) w_{l}\right\} d t
$$

Given the pumping levels of legal countries, illegal firms bind to a level of extraction that maximizes the discounted value of their payoff:

$$
\pi_{i}=\max _{w_{i}} \int_{0}^{+\infty} e^{-r t}\left\{\frac{-\beta}{2} w_{i}^{2}+\alpha w_{i}-\left(c_{2}-c_{1} H\right) w_{i}-\phi f w_{i}\right\} d t
$$

In both cases the dynamic of water table is the same:

$$
\dot{H}=\frac{1}{A S}[R+(\gamma-1) W] \quad H(0)=H_{0}
$$

The results are proposed in the following proposition 
Proposition 1 The unique stationary open-loop Nash equilibrium for the water table and the rate of extraction for legal and illegal countries are

$$
H^{*}=-\frac{R}{\mu N c_{1}(\gamma-1)}-\frac{1}{N c_{1}}\left[N \alpha-m c_{0}-(N-m)\left(\phi f+c_{2}\right)\right]
$$

where

$$
\mu=\frac{r A S}{r \beta A S-(\gamma-1) c_{1}}>0
$$

and

$$
\begin{gathered}
w_{l}^{*}=-\frac{R}{N(\gamma-1)}-\frac{\mu(N-m)\left(c_{0}-c_{2}-\phi f\right)}{N} \\
w_{i}^{*}=-\frac{R}{N(\gamma-1)}+\frac{\mu m\left(c_{0}-c_{2}-\phi f\right)}{N}
\end{gathered}
$$

Proof 1. In order to solve the problem proposed, we use the maximum principle.

Let us define the current value of the Hamiltonian $H$ in the standard way.

$$
\begin{aligned}
& \mathcal{H}_{l}=\frac{-\beta}{2} w_{l}{ }^{2}+\alpha w_{l}-\left(c_{0}-c_{1} H\right) w_{l}+\frac{\lambda_{l}}{A S}\left[R+(\gamma-1) \sum_{l=1}^{m} w_{l}+(\gamma-1) \sum_{i=m+1}^{N} w_{i}\right] l=1, \ldots, m \\
& \mathcal{H}_{i}=\frac{-\beta}{2} w_{i}{ }^{2}+\alpha w_{i}-\left(c_{2}-c_{1} H\right) w_{i}-\phi f w_{i}+\frac{\lambda_{i}}{A S}\left[R+(\gamma-1) \sum_{l=1}^{m} w_{l}+(\gamma-1) \sum_{i=m+1}^{N} w_{i}\right] i=m+1, \ldots, N
\end{aligned}
$$

where $\lambda_{l}$ and $\lambda_{\mathrm{i}}$ are the adjoint variables.

We obtain the following set of necessary conditions for an interior open-loop equilibrium:

$$
\begin{gathered}
\frac{\partial \mathcal{H}_{l}}{\partial w_{l}}=0 \Longleftrightarrow-\beta w_{l}+\alpha-c_{0}+c_{1} H+\frac{\lambda_{l}}{A S}(\gamma-1)=0 \quad l=1, \ldots, m \\
\frac{\partial \mathcal{H}_{i}}{\partial w_{i}}=0 \Longleftrightarrow-\beta w_{i}+\alpha-c_{2}+c_{1} H-\phi f+\frac{\lambda_{i}}{A S}(\gamma-1)=0 \quad i=m+1, \ldots, N
\end{gathered}
$$

which give us: 


$$
\begin{array}{cc}
w_{l}=\frac{1}{\beta}\left[\alpha-c_{0}+c_{1} H+\frac{\lambda_{l}(\gamma-1)}{A S}\right] \quad l=1, \ldots, m \\
w_{i}=\frac{1}{\beta}\left[\alpha-c_{2}+c_{1} H-\phi f+\frac{\lambda_{i}(\gamma-1)}{A S}\right] \quad i=m+1, \ldots, N
\end{array}
$$

The adjoint equations are:

$$
\begin{gathered}
\dot{\lambda}_{l}=r \lambda_{l}-c_{1} w_{l} \quad l=1, \ldots, m \\
\dot{\lambda}_{i}=r \lambda_{i}-c_{1} w_{i} \quad i=m+1, \ldots, N
\end{gathered}
$$

and the trasversality conditions being:

$$
\begin{gathered}
\lim _{t \rightarrow+\infty} e^{-r t} \lambda_{l}(t) \geq 0 \quad \lim _{t \rightarrow+\infty} e^{-r t} \lambda_{l}(t) H(t)=0 \quad l=1, \ldots, m \\
\lim _{t \rightarrow+\infty} e^{-r t} \lambda_{i}(t) \geq 0 \quad \lim _{t \rightarrow+\infty} e^{-r t} \lambda_{i}(t) H(t)=0 \quad i=m+1, \ldots, N
\end{gathered}
$$

Taking into account that, at the steady state

$$
\dot{H}=\dot{\lambda}=0
$$

the first order conditions and the adjoint equations are used to determine the stationary equilibrium :

$$
\begin{gathered}
\dot{\lambda}_{l}=0 \Longleftrightarrow \lambda_{l}=\frac{c_{1} w_{l}}{r} \Longleftrightarrow \lambda_{l}=\frac{c_{1} A S}{r \beta A S-c_{1}(\gamma-1)}\left(\alpha-c_{0}+c_{1} H\right) \quad l=1, \ldots, m \\
\dot{\lambda}_{i}=0 \Longleftrightarrow \lambda_{i}=\frac{c_{1} w_{i}}{r} \Longleftrightarrow \lambda_{i}=\frac{c_{1} A S}{r \beta A S-c_{1}(\gamma-1)}\left(\alpha-c_{2}+c_{1} H-\phi f\right) \quad i=m+1, \ldots, N
\end{gathered}
$$

and moreover

$$
\dot{\lambda_{l}}=0 \Longleftrightarrow w_{l}=\frac{r \lambda_{l}}{c_{1}} \quad \text { and } \quad \dot{\lambda_{i}}=0 \Longleftrightarrow w_{i}=\frac{r \lambda_{i}}{c_{1}}
$$

From differential equation (2) we have

$$
\dot{H}=0 \Longleftrightarrow R+m(\gamma-1) w_{l}+(N-m)(\gamma-1) w_{i}=0
$$


By substitution of $w_{l}$ and $w_{i}$, we obtain the steady state value of water table given by equation (3) and the rate of extraction for legal firms (equation(5)) and for illegal ones (equation (6)).

The stationary open-loop Nash equilibrium depends on the number of firms that exploit the aquifer, on the number of legal and illegal firms and on the parameters that characterize penalties.

Now, we consider the system composed by the adjoint equations and the dynamic of the water table

$$
\left\{\begin{array}{l}
\dot{\lambda_{l}}=r \lambda_{l}-c_{1} w_{l} \\
\dot{\lambda_{i}}=r \lambda_{i}-c_{1} w_{i} \\
\dot{H}=\frac{1}{A S}\left[R+(\gamma-1) \sum_{l=1}^{m} w_{1}+(\gamma-1) \sum_{i=m+1}^{N} w_{i}\right]
\end{array}\right.
$$

Substituting (7) and (8) we obtain the following system

$$
\left\{\begin{array}{l}
\dot{\lambda_{l}}=q \lambda_{l}+l H+L \\
\dot{\lambda_{i}}=q \lambda_{i}+l H+Q \\
\dot{H}=s \lambda_{l}+v \lambda_{i}+H z+M
\end{array}\right.
$$

where

$$
\begin{aligned}
& q=\frac{r \beta A S-c_{1}(\gamma-1)}{\beta A S}>0, \quad l=-\frac{c_{1}^{2}}{\beta}<0, L=-\frac{c_{1}\left(\alpha-c_{0}\right)}{\beta}, \quad s=\frac{m(\gamma-1)^{2}}{\beta(A S)^{2}}>0 \\
& Q=-\frac{c_{1}\left(\alpha-c_{2}-\phi f\right)}{\beta}, \quad v=\frac{(N-m)(\gamma-1)^{2}}{\beta(A S)^{2}}>0, \quad z=\frac{c_{1} N(\gamma-1)}{\beta A S}<0 \\
& M=\frac{R \beta+N(\gamma-1) \alpha-(\gamma-1)\left[m c_{0}+(N-m)\left(c_{2}+\phi f\right)\right]}{\beta A S}
\end{aligned}
$$

The equilibrium point of the system is

$$
\bar{H}=-\frac{Q v+s L-M q}{s l+v l-z q}, \quad \bar{\lambda}_{l}=-\frac{M q l+L v l-L q z-Q v l}{q(s l+v l-z q)}, \bar{\lambda}_{i}=-\frac{M q l+s l Q-s l L-z q Q}{q(s l+v l-z q)}
$$

Which is the same obtained in Proposition 1 . The stability properties of the stationary state 


$$
\left(\bar{H}, \overline{\lambda_{l}}, \bar{\lambda}_{i}\right)
$$

can be analyzed evaluating the following associated Jacobian matrix:

$$
J\left(H, \lambda_{l}, \lambda_{j}\right)=\left(\begin{array}{ccc}
q & 0 & l \\
0 & q & l \\
s & v & z
\end{array}\right)
$$

We have that the determinant of the Jacobian matrix of the system of differential equations is negative

$$
\operatorname{Det} J\left(\bar{H}, \overline{\lambda_{l}}, \bar{\lambda}_{i}\right)=q[q z-l(s+v)]=\frac{N(\gamma-1) c_{1} r}{\beta A S}<0
$$

These results imply that the steady state of the system is a saddle point and that there exists an optimal path which leads to it.

The roots of the characteristic equation associated with the homogeneous part of the system are three, are all real and are two positive and one negative. The negative root is

$$
x=\frac{-\left[(\gamma-1)(1-N) c_{1}-r \beta A S\right]-\sqrt{\left[(\gamma-1)(1-N) c_{1}-r \beta A S\right]^{2}-4 \beta A S(\gamma-1) r c_{1} N}}{2 \beta A S}
$$

Then, using the trasversality conditions to select the stable solution and the initial water table $H(0)=H_{0}$, to calculate the coeffcient of the solution, the following path

$$
H(t)=H^{*}+\left(H_{0}-H^{*}\right) e^{x t}
$$

is the open-loop Nash equilibrium path. The equilibrium water table path converges to the stationary equilibrium water table, which does not depend on the initial water table.

\subsection{Comparative Statics}

In this Section we propose an analysis of the steady state $\left(H, w_{l}, w_{i}\right)=\left(H^{*}, w^{*}, w^{*}{ }_{i}\right)$ when the parameters of the model change. Table 1 shows the results of this analysis. The parameters effects have been estimated from the partial derivatives of the equilibrium coordinates $\mathrm{H}^{*}$, $\mathrm{W}^{*}{ }_{1}$ and $w^{*}{ }_{i}$.

First of all it is possible to see that if the number of countries $N$ that exploit the resource increases, then the steady state of water table and the rate of extraction, both for legal and for illegal countries, can increase or decrease. The same result we have studying the partial derivative of the steady state respect to the number $m$ of legal countries, in fact it is possible that it is positive or negative. 
In relation to parameters $f$ and $\phi$ that characterize the punishment of illegal countries, we see that if they increase than the height of the water as well as the rate of extraction of legal countries increase while the rate of illegal countries decreases.

About the natural recharge, the equilibrium coordinates increase, instead with respect the area of aquifer $A S$, the share of $H$ decreases while the fitness of $w_{l}$ and $w_{i}$ decreases or increases. About the fixed costs $c_{0}$ and $c_{2}$ the height of the water always increases instead the rates of extraction can increase or decrease, about the parameter $c_{1}$ all the components of the equilibrium can change.

\begin{tabular}{c|r|r|r}
\hline & $H^{*}$ & $w_{l}^{*}$ & $w_{i}^{*}$ \\
\hline \hline$\nearrow N$ & $\searrow \nearrow$ & $\searrow \nearrow$ & $\searrow \nearrow$ \\
\hline$\nearrow m$ & $\searrow \nearrow$ & $\searrow \nearrow$ & $\searrow \nearrow$ \\
\hline$\nearrow f$ & $\nearrow$ & $\nearrow$ & $\searrow$ \\
\hline$\nearrow \phi$ & $\nearrow$ & $\nearrow$ & $\searrow$ \\
\hline$\nearrow R$ & $\nearrow$ & $\nearrow$ & $\nearrow$ \\
\hline$\nearrow \alpha$ & $\searrow$ & 0 & 0 \\
\hline$\nearrow \beta$ & $\nearrow$ & $\nearrow \searrow$ & $\nearrow \searrow$ \\
\hline$\nearrow r$ & $\searrow$ & $\searrow \nearrow$ & $\searrow \nearrow$ \\
\hline$\nearrow c_{0}$ & $\nearrow$ & $\searrow$ & $\nearrow$ \\
\hline$\nearrow c_{1}$ & $\searrow \nearrow$ & $\searrow \nearrow$ & $\searrow \nearrow$ \\
\hline$\nearrow c_{2}$ & $\nearrow$ & $\nearrow$ & $\searrow$ \\
\hline$\nearrow A S$ & $\searrow$ & $\searrow \nearrow$ & $\searrow \nearrow$ \\
\hline \hline
\end{tabular}

\section{Concluding remarks}

In this paper, we have extended the literature quoted, examining the presence of legal and illegal firms in a common groundwater resource exploitation. In the literature, numerous papers have studied groundwater exploitation, in particular, examining only the socially optimal exploitation or the private exploitation of the aquifer and they have concluded that private exploitation is inefficient, in terms of stock and welfare, in comparison to optimal exploitation.

This ineffciency is due to the various externalities but never they include the possibility that firms can be legal or illegal. Illegal water use is a key issue to understand many of the problems related to depleting and overexploited stocks. Empirical evidence demonstrates that illegal activities, including unlicensed water extraction, illegal logging and pollution discharges, are at the root of the degradation of water-related habitats.

So, our aim is to consider $N$ countries that differ in terms of choice of their behavior, legal or illegal. We have shown as a the dynamic game, the number of legal and illegal firms affect the steady states of the water table and the rate of extraction for the two types of behavior. In particular, we have proposed a comparative statics analysis with respect to all parameters which characterize the model underlying the rules of penalties. 


\section{References}

Biancardi, M. and L. Maddalena (2018). Competition and cooperation in the exploitation of the groundwater resource. Decision in Economics and Finance. 41 (2), 219-237,2018.

Brown, G.M. (2000). Renewable natural resource management and use without markets. Journal of Economic Literature, Vol. 38(4), 875914.

Burke, J. (2003). Groundwater for irrigation: productivity gains and the need to manage hydroenvironmental risk. Llamas, M. R. and Custodio, E., editors, Intensive Use of Groundwater. Challenges and Opportunities, chapter 3, Taylor and Francis, Vol. 59-80.

Esteban, E. and J. Albiac, (2011). Groundwater and ecosystems damages: Questioning the Gisser-Sanchez effect. Ecological Economics, Vol. 70, 2062-2069.

Gisser, M. and D.A. Sanchez, (1980). Competition versus optimal control in groundwater pumping. Water Resources Research, Vol. 16, 638-642.

Koundouri, P. (2004). Current issues in the economics of groundwater resource manage-ment. Journal of Economic Surveys, Vol. 18(5), 703-740.

Koundouri, P. and A. Xepapadeas, (2004). Estimating accounting price for common pool natural resources: a distance function approach. Water Resources Research, Vol. 40(6).

Negri, D.H. (1989). The common property aquifer as a differential game. Water Resources Research, Vol. 25, 9-15.

Provencher, B. and O. Burt, (1993). The externalities associated with the Common Property Exploitation of groundwater. Journal of Environmental Economics and Management, Vol. 24, 139-158.

Rubio, S. J. and B. Casino, (2001). Competitive versus efficient extraction of a common property resource. The groundwater case. Journal of Economics Dynamics and Control, Vol. 25, 1117-11137.

Rubio, S. J. and B. Casino, (2003). Strategic Behavior and efficiency in the common prop-erty extraction of groundwater. Environmental and Resource Economics, Vol. 26, 73-87. 